\title{
A NONDEVELOPABLE ČECH-COMPLETE SPACE WITH A POINT-COUNTABLE BASE
}

\author{
S. W. DAVIS
}

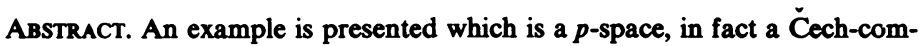
plete space, which has a point-countable base and is not developable. This answers questions raised by Burke in 1970 and by Burke and Tall in 1972.
\end{abstract}

1. Introduction and definitions. The notion of Čech-completeness was de-

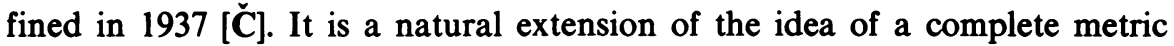
space, and is simply that a space is Čech-complete if it is a $G_{\delta}$-set in its Stone-Čech compactification. For the purposes of this note another characterization will be useful. This is essentially the form found by Frolik in 1960 [F], and is the definition we shall use.

Definition $1.1[\check{\mathbf{C}}],[\mathbf{F}]$. A completely regular $T_{2}$ space $X$ is Čech-complete if and only if there is a sequence $\left\langle\mathcal{G}_{n}: n \in \omega\right\rangle$ of open covers of $X$ such that if $\mathcal{F}$ is a collection of closed subsets of $X$ with finite intersection property and for each $n \in \omega$ there is $F_{n} \in \mathcal{F}$ and $G_{n} \in \mathcal{G}_{n}$ with $F_{n} \subseteq G_{n}$, then $\cap \mathscr{F} \neq \varnothing$.

The class of $p$-spaces was introduced in 1963 by Arhangel'skii [ $\mathbf{A}_{1}$ ] as a class containing both the metrizable spaces and the locally compact spaces. The $p$-spaces, and relatives like the strict $p$-spaces $\left[\mathbf{A}_{1}\right]$ and $w \Delta$-spaces [Bo], have been among the most fruitful and thoroughly investigated classes of spaces in general topology. The authors of papers concerning these spaces are far too numerous to recite here.

Definition $1.2\left[\mathbf{A}_{1}\right.$ ]. A completely regular $T_{2}$ space $X$ is called a $p$-space if in the Stone-Čech compactification $\beta X$ there is a sequence $\left\langle\gamma_{n}: n \in \omega\right\rangle$ of open covers of $X$ such that $\bigcap_{n \in \omega} \operatorname{st}\left(x, \gamma_{n}\right) \subseteq X$ for each $x \in X$.

Since a locally compact $T_{2}$ space is open in its Stone-Čech compactification, it is clear that every locally compact $T_{2}$ space is Čech-complete and that every Cech-complete space is a $p$-space.

The questions which we will answer in this note were raised by Burke [B] in 1970 and by Burke and Tall [BT] at the Pittsburgh conference in 1972.

QUESTION 1.3 [B]. Is a $p$-space with a point-countable base a Moore space?

QUESTION 1.4 [BT]. If $X$ is a Čech-complete space having a point-countable base, must $X$ be developable?

Motivation for these questions is contained in the following theorems.

THEOREM $1.5[\mathrm{CM}]$. A locally compact $T_{2}$ space $X$ with a point-countable base is metrizable.

Presented to the Society, November 4, 1978; received by the editors March 21979.

AMS (MOS) subject classifications (1970). Primary 54G20; Secondary 54E99, 54E30, 54D45. 
THEOREM 1.6 [B], [ $\left.\mathbf{H}_{2}\right]$. A metacompact p-space with a point-countable base is a Moore space.

THEOREM 1.7 [B]. A subparacompact p-space with a point-countable base is a Moore space.

In this note we show that both Questions 1.3 and 1.4 have negative answers by exhibiting a Čech-complete space with a $\sigma$-locally countable and $\sigma$-disjoint base which is neither perfect nor $\theta$-refinable. It is interesting to compare the covering properties in the example with Burke's theorems. Having a $\sigma$-disjoint base, the example is screenable (thus weakly $\theta$-refinable) which implies metacompact for developable spaces $\left[\mathbf{H}_{1}\right]$ and implies subparacompact for perfect spaces [BL]. This shows that there is very little room for improvement in these theorems.

In our set theoretic usage, we will follow the custom that cardinal numbers are initial ordinals, and we use $c$ to denote the cardinality of the reals, i.e. $c=2^{\omega}$.

2. The example. Before launching into the technical details of the construction, we feel it will be helpful to give a brief intuitive description of the example. We begin with a zero-dimensional, nonseparable metric space $M$, the so-called "Baire space of weight $c$ ". To this we attach a closed discrete set $F$ of cardinality $c$. Neighborhoods of points of $F$ will be tails of carefully selected discrete sequences of metric balls in $M$. The critical property of the sequences, in proving Čech-completeness, is that no two terms of a sequence can intersect the same term of another sequence.

EXAMPLE 2.1 There is a zero-dimensional $T_{2}$ space $Z$ which is Čech-complete, has a $\sigma$-locally countable and $\sigma$-disjoint base (hence, a point-countable base), but is not developable.

Proof. Let $D$ be a discrete space of cardinality $c$, let $M=\prod_{k \in \omega} D_{k}$, where $D_{k}=D$ for each $k \in \omega$, and let $F=\{\alpha: \alpha<c\}$. For each point $x \in \prod_{i=0}^{k} D_{i}$, we denote by $[x]$ the basic open set in $M$ which is given by $\left\{z: \pi_{i} z=\pi_{i} x\right.$ for $i<k\}$. Let $\mathfrak{K}_{k}=\left\{[x]: x \in \Pi_{i=0}^{k} D_{i}\right\}$ and let $\left\{\delta_{\alpha}: \alpha<c\right\}$ be a well ordering of all countable subsets of $\cup_{k \in \omega} \Re_{k}$ such that each $\delta_{\alpha}$ is contained in some $\mathfrak{T}_{k(\alpha)}$, and the projection of $\delta_{\alpha}$ into $D_{0}$ is one-to-one. We define, by induction on $\alpha$, sequences $s_{\alpha}: \omega \rightarrow \cup \delta_{\alpha}$ and $B_{\alpha}: \omega \rightarrow \cup_{k \in \omega} \Re_{k}$ such that the following are true for each $\alpha<c$ :

(1) If $i \neq j, s_{\alpha}(i)$ and $s_{\alpha}(j)$ are not in the same element of $\delta_{\alpha}$.

(2) $s_{\alpha}(n) \in B_{\alpha}(n)$.

(3) $B_{\alpha}(n) \in \cup \bigcup_{i>n+k(\alpha)} \mathfrak{M}_{i}$.

(4) If $\alpha<\beta$, then $\left(\cup_{n \in \omega} B_{\beta}(n)\right) \cap\left\{s_{\alpha}(k): k \in \omega\right\}=\varnothing$, and

$$
\left(\bigcup\left\{B_{\alpha}(n): B_{\alpha}(n) \in \bigcup_{i>k(\beta)+1} \Re_{i}\right\}\right) \cap\left(\bigcup_{n \in \omega} B_{\beta}(n)\right)=\varnothing
$$

(5) If $B_{\beta}(n)=\left[x_{1}, x_{2}, \ldots, x_{k(\beta)}, \ldots, x_{m_{n}}\right]$, then $\left[x_{1}, x_{2}, \ldots, x_{k(\beta)+1}\right] \cap$ $B_{\alpha}(n)=\varnothing$ whenever $\alpha<\beta$ and $B_{\alpha}(n) \in \bigcup_{i>k(\beta)+1} \Re_{i}$. 
The space $Z=M \cup F$, and $M$ is an open subspace. For $\alpha \in F$, we define $U_{n}(\alpha)=\{\alpha\} \cup\left(\cup_{k>n} B_{\alpha}(k)\right)$. The collection $\left\{U_{n}(\alpha): n \in \omega\right\}$ will be an open neighborood base at $\alpha$. It is easy to see that this topology on $Z$ is a zero-dimensional $T_{2}$ space, hence $Z$ is completely regular.

We now show that $Z$ is Čech-complete. For $x \in M$, we let $U_{n}(x)=$ $\left[x_{1}, x_{2}, \ldots, x_{n}\right] \in \mathfrak{N}_{n}$, for each $n \in \mathfrak{N}$. Note that with the usual metric on $M, U_{n}(x)=B\left(x, 2^{-n}\right)$. For $n \in \omega$ let $\mathcal{S}_{n}=\left\{U_{n}(z): z \in Z\right\}$. It is clear that $\mathcal{S}_{n}$ is an open cover of $Z$ for each $n \in \omega$. Suppose $\mathscr{F}$ is a collection of closed subsets of $Z$ with finite intersection property and for each $n \in \omega$ there is $F_{n} \in \mathscr{F}$ and $G_{n} \in \mathcal{G}_{n}$ with $F_{n} \subseteq G_{n}$. We let $H_{n}=\bigcap_{i<n} F_{i}$ for each $n \in \omega$. First, if there exists $k \in \omega$ with $G_{k}=U_{k}(x)$ with $x \in M$, then $H_{n} \subseteq U_{n}\left(x_{n}\right)$ for some $x_{n} \in M$ for each $n \geqslant k$, since $H_{n} \subseteq G_{n} \cap U_{k}(x)$ and if $G_{n}=U_{n}(\alpha)$ $=\{\alpha\} \cup\left(\cup_{i>n} B_{\alpha}(i)\right)$ there is exactly one $i \geqslant n$ such that $B_{\alpha}(i) \cap U_{k}(x) \neq$ $\varnothing$, namely the one for which $\pi_{0} B_{\alpha}(i)=\pi_{0} x$, and $B_{\alpha}(i) \in \Re_{j}$ for some $j>n$. In this case, by the completeness of $M, \cap_{n \in \omega} H_{n} \neq \varnothing$ and, by choice of $\left\langle\mathcal{S}_{n}\right.$ : $n \in \omega\rangle$, is in fact a single point, say $z$. If there is $H \in \mathscr{F}$ with $z \notin H$, we choose $n \in \omega$ such that $U_{n}(z) \cap H=\varnothing$, then $H_{n+1} \cap H=\varnothing$ which contradicts the finite intersection property. Now suppose $G_{n}=U_{n}\left(\alpha_{n}\right)$ with $\alpha_{n} \in F$ for each $n \in \omega$. If $H_{n} \cap F \neq \varnothing$ for every $n \in \omega$, then there is $\alpha \in F$ such that $\alpha_{n}=\alpha$ for every $n \in \omega$ and $\{\alpha\}=\bigcap_{n \in \omega} H_{n}$. If there is $H \in \mathscr{F}$ with $\alpha \notin H$, then we choose $n \in \omega$ such that $U_{n}(\alpha) \cap H=\varnothing$, and we have $H_{n} \cap H=\varnothing$ which contradicts the finite intersection property. Finally, suppose $H_{n} \cap F=\varnothing$ for some $n \in \omega$, then for each $\alpha \in F$ there exists $k \in \omega$ such that $U_{k}(\alpha) \cap H_{n}=\varnothing$ and $k \geqslant n$. Hence $U_{k}(\alpha) \cap H_{k}=\varnothing$. So we have that $\left\{\alpha_{n}: n \in \omega\right\}$ is an infinite set. Pick $\alpha_{n_{0}}, \alpha_{n_{1}}$ distinct members of $\left\{\alpha_{n}\right.$ : $n \in \omega\}$. We may assume $\alpha_{n_{0}}>\alpha_{n_{1}}$. The set $U_{n_{1}}\left(\alpha_{n_{1}}\right) \cap U_{n_{0}}\left(\alpha_{n_{0}}\right)$ is a disjoint union of not more than $k\left(\alpha_{n_{0}}\right)$ balls in $M$ each having radius less than $2^{-m}$, where $m=\max \left\{n_{0}, n_{1}\right\}$, by (3) and (4). Moreover, for $\alpha, \beta \in F$, no two terms of $B_{\alpha}$ can intersect the same term of $B_{\beta}$ by (1), (2) and choice of $\left\{\delta_{\alpha}\right.$ : $\alpha<c\}$. By the completeness of $M, \cap_{n \in \omega} G_{n}=\left\{z_{i}: 0<i \leqslant k \leqslant k\left(\alpha_{n_{0}}\right)\right\}$. Suppose there is a set $A_{i} \in \mathscr{F}$ with $z_{i} \notin A_{i}$ for each $i<k$. We may choose $n \in \omega$ such that $U_{n}\left(z_{i}\right) \cap \cap{ }_{i<k} A_{i}=\varnothing$ for each $i \leqslant k$. Now $G_{n} \subseteq$ $\cup_{i<k} U_{n}\left(z_{i}\right)$, so we have that $H_{n} \cap \cap_{i<k} A_{i}=\varnothing$ which violates the finite intersection property. Thus in any case, we see that $\cap \mathscr{F} \neq \varnothing$ and the proof is complete.

This example was created by Gruenhage ([DGN, Example 3.3]) as an example of a space with a $\sigma$-locally countable base which is not developable. In [DGN], it is shown to have a $\sigma$-locally countable and $\sigma$-disjoint base. This clearly implies point-countable base. It is also shown in [DGN] that $Z$ is neither perfect nor $\theta$-refinable. Hence we see that $Z$ has the properties claimed in the title.

We list, with some redundancy, a few other properties of $Z$ which may be of interest. The space $Z$ has a base of countable order, is screenable, is meta-Lindelöf, is not para-Lindelöf, is weakly $\theta$-refinable, is a $\sigma^{\sharp}$-space, is not 
a strict $p$-space, is not a $w \Delta$-space, is not countably metacompact, is not collectionwise Hausdorff.

\section{REFERENCES}

$\mathbf{A}_{1}$. A. V. Arhangel'skii, On a class of spaces containing all metric and all locally bicompact spaces, Soviet Math. Dokl. 4 (1963), 1051-1055.

A2. _ Mappings and spaces, Russian Math. Surveys 21 (1966), 115-162.

BL. H. R. Bennett and D. J. Lutzer, $A$ note on weak $\theta$-refinablility, General Topology and Appl. 2 (1972), 49-54.

Bo. C. J. R. Borges, On metrizability of topological spaces, Canad. J. Math. 20 (1968), 795-804.

B. D. K. Burke, On p-spaces and w $\Delta$-spaces, Pacific J. Math. 35 (1970), 285-296.

BT. D. K. Burke and F. D. Tall, Problem 2, TOPO 72-General topology and its applications, Springer-Verlag, Berlin-Heidelberg-New York, 1974, p. 649.

C. E. Cech, On bicompact spaces, Ann. of Math. 38 (1937), 823-844.

CM. H. H. Corson and E. Michael, Metrizability of certain countable unions, Illinois J. Math. 8 (1964), 351-360.

DGN. S. W. Davis, G. Gruenhage and P. J. Nyikos, $G_{8}$-sets in symmetrizable and related spaces, General Topology and Appl. 9 (1978), 253-261.

F. Z. Frolik, Generalizations of the $G_{8}$-property of complete metric spaces, Czechoslovak Math. J. 10 (1960), 359-379.

$\mathbf{H}_{1}$. R. W. Heath, Screenability, pointwise paracompactness and metrization of Moore spaces, Canad. J. Math. 16 (1964), 763-770.

$\mathbf{H}_{2}$. Semi-metrc spaces and related spaces, Topology Conference, Arizona State University, 1967, pp. 153-161.

Department of Mathematics, Miami University, Oxford, OHo 45056 